\title{
Comments
}

Comments are short papers which comment on papers of other authors previously published in the Physical Review. Each Comment should state clearly to which paper it refers and must be accompanied by a brief abstract. The same publication schedule as for regular articles is followed, and page proofs are sent to authors.

\section{Domain-growth kinetics of systems with soft walls}

\author{
Wim van Saarloos and Martin Grant* \\ AT\&T Bell Laboratories, Murray Hill, New Jersey 07974
}

(Received 25 March 1987)

\begin{abstract}
It has recently been suggested by Mouritsen on the basis of computer simulations that systems with soft domain walls exhibit slower domain growth than the $R \sim t^{1 / 2}$ growth law predicted by Lifshitz and Allen and Cahn. We underscore the reasons to believe this interpretation of the data to be incorrect, and draw attention to an experiment by Pindak, Young, Meyer, and Clark whose results are in complete agreement with the predictions of Allen and Cahn. The reason for the unexpected growth dynamics observed in Mouritsen's simulations is suggested.
\end{abstract}

After a system is rapidly quenched below its transition temperature for an order-disorder transition, the initial dynamics of the system consists of the formation of small domains of the various possible ordered phases. Soon after these domains have formed, the kinetics become dominated by the growth and shrinking, as well as merging, of these domains. ${ }^{1}$ In this regime, the typical size $R$ of the domains usually exhibits power-law growth in time, $R(t) \sim t^{n}$. The dependence of the growth exponent $n$ on the parameters of the model has recently been explored extensively with the aid of computer simulations. ${ }^{2-4}$ For nonconserved Ising systems, the ordering dynamics is dominated by the curvature-driven motion of domain walls discussed by Turnbull, ${ }^{5}$ Lifshitz, $^{6}$ and Allen and Cahn, ${ }^{7}$ and this yields ${ }^{6} n=\frac{1}{2}$. If the number of ground states becomes larger than 2, topological constraints can, however, slow down the growth; such behavior has, for example, been seen in simulations ${ }^{3}$ of $q$-state Potts models, where $n$ is found to be smaller than $\frac{1}{2}$ and to decrease with $q$. Recently, it has been claimed ${ }^{8,9}$ that softness of domain walls can also slow down the growth kinetics with respect to that of the Ising model. This suggestion was based on the results of computer simulations ${ }^{8,9}$ which appeared to indicate that a growth exponent $n \simeq \frac{1}{4}$ is a general feature of soft-wall models, not only of those with more than two ground states, but also of those with only two ground states.

The idea that the softness of the domain walls alone would affect the growth kinetics is rather surprising, since there is no particular reason why the driving force for the motion of a soft wall would not be linear in its curvature, as obtained in all approaches. ${ }^{5-7}$ In other words, in systems with a twofold-degenerate ground state so that topological constraints are not the rate-limiting factor, one ex- pects the Lifshitz-Allen-Cahn-type growth law, with $n=\frac{1}{2}$, to hold, provided the width of the walls is much smaller than their radius of curvature. ${ }^{10,11}$ This is precisely what Milchev, Binder, and Heermann ${ }^{11}$ found in their simulations of the $\phi^{4}$ continuous-spin Ising model. Moreover, a critical assessment of the interpretation of Mouritsen's Monte Carlo data has been given by Kaski, Kumar, Gunton, and Rikvold, ${ }^{8}$ who argued that a better interpretation of the data was consistent with $n=\frac{1}{2}$. Finally, Pindak, Young, Meyer, and Clark ${ }^{12}$ measured $n=\frac{1}{2}$ in an experiment on liquid crystals, whose relevance appears to have been overlooked in this field.

In view of this apparent discrepancy concerning the relevance of the softness of the walls, we investigate some of the possible reasons that the model studied most recently by Mouritsen ${ }^{9}$ gives rise to slower growth dependence than expected. The reason we concentrate only on this model is that it is simple enough that we can analyze some of its aspects analytically. We will show that in contrast to what has been asserted, this particular model actually does not have soft walls in the parameter range explored in the computer simulations. Moreover, we will argue that the small effective growth exponent found by Mouritsen $^{9}$ may be ascribed to effects that are specific to the particular zero-temperature Monte Carlo quench method employed; in particular, we argue that for long times the domain size grows as $(\ln t)^{1 / 2}(n=0)$ in the limit in which the system becomes one dimensional, obtained by letting the next-nearest-neighbor interaction vanish. At finite temperatures, these effects may give rise to slow crossover, but we do expect the model to exhibit $t^{1 / 2}$ growth dynamics at long times and finite temperatures. Before presenting these results, however, we will first try to put the theory and the experiments by Pindak et al. ${ }^{12}$ into per- 
spective.

Lifshitz $^{6}$ and Turnbull $^{5}$ (LT) as well as Allen and $\mathrm{Cahn}^{7}$ (AC) all argue that the normal growth rate $V_{n}$ of an interface is proportional to its curvature $\kappa, V_{n}=c \kappa$. For the radius $R$ of a shrinking droplet this yields $d R / d t=-2 c / R \quad(c / R$ in two dimensions), and so $R^{2}=2 c\left(t_{0}-t\right)$ where $t_{0}$ is the time at which the droplet disappears. This shows that $n=\frac{1}{2}$. AC stress, however, that their prediction for the coefficient $c$ is different from that of LT. The latter argue that $c=\mu \sigma$, with $\mu$ a mobility and $\sigma$ the surface tension of the interface, while in AC's squared-gradient theory $c$ is equal to the prefactor of the gradient term in the free energy. The origin of this discrepancy can be summarized as follows. In all approaches the driving force for the motion of the interface is the excess energy associated with the interface, which is proportional to the surface tension. The physical picture on which LT base their discussion of the resulting curvature-driven dynamics is that of a sharp interface in an alloy, in which the rate of motion of the interface is determined by the diffusion (exchange) of atoms at the boundary. ${ }^{6}$ This yields a relation of the form $c=\mu \sigma$ with $\mu$ a mobility coefficient for this process. AC, on the other hand, consider interfaces whose structure is smoothly varying in the direction normal to the interface ("soft"). In a squared gradient theory of the type considered by AC, the width of such a continuous interface is inversely proportional to the surface tension. When such an interface moves, the rate at which the order parameter changes in the interface region is inversely proportional to the width of the interface, so that both the driving force and the energy dissipation are inversely proportional to the interface width. As a result, within the AC theory, the normal growth rate coefficient $c$ becomes independent of the interface width, ${ }^{13}$ and hence $\sigma$.

Obviously, the conclusion from both approaches is that the sharpness or softness of the walls does not affect the growth law, as one would expect: $n=\frac{1}{2}$ in both cases. Only the prefactor $c$ will, in general, depend on the structure of the walls, although for sufficiently wide walls described by the AC theory, the rate coefficient $c$ becomes independent of the wall width. These results are clearly at variance with Mouritsen's claims about the softness of the walls.

Many of the AC results were rederived independently by Pindak et al., ${ }^{12}$ who performed experiments on orientation patterns in freely suspended smectic- $C$ liquid-crystal films. Since smectic- $C$ films have a permanent electric dipole moment $\mathbf{P}$, the director angle $\phi$ can be oriented with an electric field $E$ and the free energy of a field becomes in the one-constant approximation ${ }^{14}$

$$
F=\int d x \int d y\left[\frac{1}{2} K(\nabla \phi)^{2}-P E \cos \phi\right]
$$

After turning on the field $\mathbf{E}$, the director field in the experiment reorganizes into domains of the lowest free-energy state $\phi=0, \pm 2 \pi, \ldots$. The domains are separated by $2 \pi$ disclination lines which either end at point defects or form closed loops. The radius $R$ of the latter "droplets" was found ${ }^{12}$ to shrink as $R^{2}=c\left(t_{0}-t\right)$, with $c$ independent of the wall width, which in agreement with Eq. (1) was found to vary as $E^{-1 / 2}$. Thus, these experiments provide a direct verification of the AC predictions that both the growth exponent $n$ and the rate parameter $c$ are independent of the "softness" of the walls. Since these liquidcrystal films provide such a clean realization of nonconserved ordering dynamics, we hope that these experiments will be pursued in more detail to measure the structure function $S(q, t)$, for which, to our knowledge, the theoretical predictions have not been tested extensively.

We now turn to the two-dimensional lattice model recently investigated by Mouritsen. ${ }^{9}$ The Hamiltonian for the angle variables $\phi_{i}$ of this model can be written as ${ }^{15}$

$$
H=-J \sum_{\mathrm{nnn}} \cos \left(\phi_{i}-\phi_{j}\right)-\sum_{\substack{i, j \\ i>j}}^{x} \cos \phi_{i} \cos \phi_{j} .
$$

The first sum is over next-nearest-neighbor terms on the square lattice, while the second term is over nearest neighbors in the horizontal $(x)$ direction only [see Figs. 1(a)-1(c)]. We will take $\phi=0$ when the spins are pointing up [see Fig. 1(c)], so that in the two ground states of the system correspond to the spins pointing up or down.

The width of walls in this model depends on $J$, larger values of $J$ giving rise to wider walls. Mouritsen ${ }^{9}$ presents data for $J=0.2$, reporting that data in the range

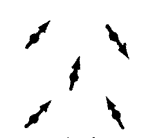

(a)

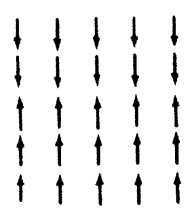

(d)

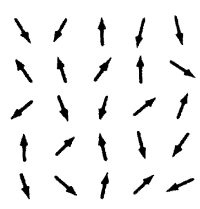

(f)

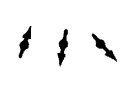

(b)

$$
\begin{aligned}
& 11-11 \\
& 11-11 \\
& 11-11 \\
& 11-1 \mid \\
& 11-1 \mid
\end{aligned}
$$

(e)

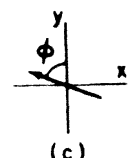

(c)

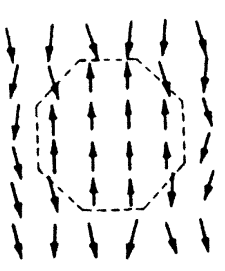

(n)
FIG. 1. (a) The spin in the center is coupled through ferromagnetic next-nearest-neighbor interaction to four other spins. (b) The nearest-neighbor interaction only couples spins within the same row. (c) Convention for the angle $\phi$ and the $x$ and $y$ directions. (d) For $J<1$, a wall parallel to the $x$ direction is hard in that all spins are at angles 0 or $\pi$. (e) A symmetric wall parallel to the $y$ direction. In the two rows adjacent to the center one the spins are rotated by about $9.5^{\circ}$ for $J=0.2$. (f) For small $J$, the spins rearrange quickly after a quench into domains of up and down spins. (g) In the $J=0$ limit, the model becomes one-dimensional because rows get decoupled. The spin labeled $\boldsymbol{A}$ separates up and down domains. (h) A domain of perfectly aligned $(\phi=0)$ up spins in a sea of down spins is frozen in, in spite of the $J$ interaction. 
$0.02<J<2$ yield similar results. For these values, however, the walls are quite sharp: From a gradient expansion of the Hamiltonian (2), assuming that the $\phi_{i}$ vary gradually, one finds that the wall width (in units of the lattice parameter) is of order $\sqrt{J}$. In the range of parameter values investigated, this is of order unity or smaller, indicating that the walls are actually quite localized. Of course, this analysis is, strictly speaking, inconsistent, since the assumption that the $\phi_{i}$ vary slowly breaks down in this regime. We have therefore performed a more careful analysis of two types of walls. For the zerotemperature walls normal to the $y$ direction, we find that these are sharp for $J<1$, i.e., in the ground state the $\phi_{i}$ change abruptly from 0 to $\pi$ from one row to the next, as illustrated in Fig. $1(\mathrm{~d})$. (For $J \gtrsim 1$, the spins get rotated slightly near the wall. ${ }^{16}$ ) The structure of the walls normal to the $x$ direction is more complicated. For angles $\phi_{i}$ varying only in the $x$ direction (all $\phi_{i}$ are the same in each column), the condition $\partial H / \partial \phi_{i}=0$ for minima of the energy can, with the aid of some trigonometric relations, be written as

$$
\begin{array}{r}
2 J \cos \left(\frac{\phi_{i+1}-\phi_{i-1}}{2}\right)[ \\
\left.-\cos \phi_{i} \sin \left(\frac{\phi_{i+1}+\phi_{i-1}}{2}\right)\right]=0 .
\end{array}
$$

This equation allows sharp-wall solutions of which the angles $\phi$ abruptly change from 0 to $\pi$ at some $i$, but these do not have the lowest energy. A lower-energy solution is, for example, the symmetric wall for which at row $i=0$, say, $\phi_{0}=\pi / 2$, and $\phi_{1}=\pi-\phi_{-1}$. Assuming that $\phi_{i} \rightarrow 0$ for large negative values of $i$, we then find from Eq. (3), evaluated at $i=-1$,

$$
\begin{aligned}
\phi-1= & \arctan \left(\frac{\sin (\pi / 4+\phi-2 / 2)}{\cos (\pi / 4+\phi-2 / 2)} \frac{1}{1+J^{-1}}\right) \\
& =\arctan \left(\frac{J}{1+J}\right),
\end{aligned}
$$

where we used the fact that $\phi_{-2}$ can be neglected for $J \lesssim 1$. This formula shows that the spins adjacent to the one at $i=0$ only turn slightly for $J \lesssim 1-$ e.g., for $J=0.2$, the value used by Mouritsen, we have $\phi_{-1}=9.5^{\circ}$, and the wall looks like the one sketched in Fig. 1(e).

These considerations show that contrary to what has been suggested, the lattice model [Eq. (1)] does not have continuous, soft walls like those of $\mathrm{AC}$ in the regime investigated by Mouritsen (screening of soft walls has been suggested $^{8}$ as the reason for dynamics slower than $t^{1 / 2}$ ). Why, then, did the simulation not exhibit a growth exponent $n=\frac{1}{2}$ ? We suggest that this is due to the particular zero-temperature Monte Carlo method employed in the study. In these zero-temperature quenches the orientation of a spin is only changed to a new randomly generated angle if this results in a lower energy. For quenches of systems with small $J$, the second term in the energy dominates and since this term strongly favors the alignment of spins in either the up or down state, the ini- tial evolution of the system will be dominated by the grouping of the spins into domains of mainly up $(-\pi / 2<\phi<\pi / 2)$ or down $(\pi / 2<\phi<3 \pi / 2)$ spins, as indicated in Fig. 1(f). As time progresses, the spins in the bulk of the domains continue to relax towards the ground state $\phi=0$ or $\phi=\pi$. The more a spin has relaxed towards the $\phi=0$ or $\phi=\pi$ state before this spin becomes part of the boundary of a domain, the more unlikely it will be that such a spin is flipped in a Monte Carlo step; in other words, the chance that a random attempt to change a spin angle lowers the energy becomes smaller and smaller as long as the next nearest-neighbor interaction is negligible. To make this explicit, we consider the model in the $J=0$ limit, so that it consists of a set of uncoupled, onedimensional rows of spins, as in Fig. $1(\mathrm{~g})$.

Consider first a spin in the bulk of this system, one whose two neighbors are pointing in the up direction, say. With the convention that the angle $\phi$ varies between $-\pi$ and $\pi$, an attempt to change this spin is accepted if the new angle is smaller (in absolute value) than the original one. Thus, in suitable time units the evolution of the distribution function $P(\phi, t)$ is given by a master equation of the form

$$
\begin{aligned}
\frac{\partial P(\phi, t)}{\partial t}= & \frac{1}{2} \int_{-\pi}^{-\phi} \phi^{\prime} P\left(\phi^{\prime}, t\right) \\
& +\frac{1}{2} \int_{\phi}^{\pi} d \phi^{\prime} P\left(\phi^{\prime}, t\right)-\frac{1}{2} \int_{-\phi}^{+\phi} d \phi^{\prime} P(\phi, t) .
\end{aligned}
$$

For long times, $P(\phi, t)$ will become more narrowly peaked around $\phi=0$, and $P(\phi, t)$ will approach a symmetric selfsimilar solution of the form $t^{\alpha} P_{s}\left(\phi t^{\alpha}\right)$ [normalization, $\int d \phi P(\phi, t)=1$, requires the presence of the factor $t^{a}$ in front of $P_{s}$ ]. To obtain the exponent $\alpha$, we note that for a symmetric distribution $[P(\phi, t)=P(-\phi, t)]$, Eq. (5) yields after differentiation

$$
\frac{\partial^{2} P}{\partial \phi \partial t}=-2 P-\phi \frac{\partial P}{\partial \phi} \text {. }
$$

Substitution of $P=t^{\alpha} P_{s}(u)$ with $u \equiv \phi t^{\alpha}$ then yields the differential equation

$$
\alpha t^{a-1}\left(u P_{s}^{\prime \prime}+2 P_{s}^{\prime}\right)=-2 P_{s}-u P_{s}^{\prime} .
$$

Clearly, in order that proper similarity solutions exist $\alpha$ must be equal to 1 ; the equation resulting for $\alpha=1$ can be solved to yield $t P_{s}(\phi t)=\frac{1}{2} t e^{-|\phi| t}$, showing that the width $\Delta \phi$ of the distribution decreases as $t^{-1}$. As a result, the probability of acceptance of a new random spin angle in this Monte Carlo method also goes down as $t^{-1}$.

This is also true for spins at the boundary of up and down domains in this one-dimensional limit. Consider, for example, the spin labeled $A$ in Fig. $1(\mathrm{~g})$. In order that the boundary between the two domains moves over by one step, this spin has to line up with one of the two domains; i.e., its angle has to fall within an interval $\Delta \phi \sim t^{-1}$ around $\phi=0$ or $\phi=\pi$. Since all angles are equally probable in this method, the probability that this happens decreases as $t^{-1}$, and so the edges between up and down domains perform a random motion with jump probabilities decreasing as $t^{-1}$. After absorbing this time dependence in a new time $\tau=\ln t$, the master equation for the position of the "interfaces" becomes equivalent to the usu- 
al one with constant jump probabilities and time variable $\tau$. Accordingly, the size $R$ of the domains grows in this limit very slowly as $R \sim \tau^{1 / 2}=(\ln t)^{1 / 2}$.

Although the situation with $J \neq 0$, but small, is more complicated, we still expect the dynamics to be affected by a similar slowing down. To illustrate this, consider the up $(\phi=0)$ domain of Fig. 1(h), which is surrounded by a sea of spins whose distribution of angles has a finite width around $\pi$. It is easy to check that none of the spins in the up domain will be flipped in the zero-temperature Monte Carlo method. For this to happen the down spins would have to point exactly down $(\phi-\pi)$. Such freezing in of perfect up domains also occurs for $J=0$ and underlies the $R \sim(\ln t)^{1 / 2}$ behavior in that limit. Hence we expect the model to be affected by very slow growth dynamics for $J \neq 0$, but small, and that the small effective exponent observed over a limited time interval in his zero-temperature simulations is due to a crossover to such a regime.

In interpreting the results of the simulations, one should also keep in mind, however, that zero-temperature dynamics can be very sensitive to the particular way in which the method is implemented. For example, if the spin angles are represented by a large but finite number of states rather than by a continuous variable, crossover to a different growth regime should eventually occur. It would therefore be of interest to examine the dynamics of the model at finite temperatures, and to reinvestigate some of the earlier work on more complicated models ${ }^{8}$ as well.

In summary, we have tried to underscore the reasons one expects the softness of the walls to have no effect on the validity of the Lifshitz-Allen-Cahn growth law, and we have drawn attention to the fact that the experiments by Pindak et al. are in full agreement with the prediction of Allen and Cahn. We have argued that Mouritsen's zero-temperature Monte Carlo method yields very slow dynamics in a simple limit of his model, and attribute the general occurrence of slow dynamics in his simulation to similar effects.

We are grateful to David A. Huse for discussions. One of us (M.G.) acknowledges support of the U.S. National Science Foundation through Grant No. DMR-83-12958.
*Present and permanent address: Department of Physics, McGill University, Montreal, Quebec, Canada H3A 2T8.

${ }^{1}$ For a review see, J. D. Gunton, M. San Miguel, and P. S. Sahni, in Phase Transitions and Critical Phenomena, edited by C. Domb and J. Lebowitz (Academic, New York, 1983), Vol. 8. References to more recent work can be found in $\mathrm{K}$. Binder, Physica A 140, 35 (1986).

2P. S. Sahni, G. Dee, J. D. Gunton, M. Phani, J. L. Lebowitz, and M. Kalos, Phys. Rev. B 24, 410 (1981); K. Kaski, M. C. Yalabik, J. D. Gunton, and P. S. Sahni, ibid. 28, 5263 (1983); E. T. Gawlinski, M. Grant, J. D. Gunton, and K. Kaski, ibid. 31, 281 (1985).

${ }^{3}$ S. A. Safran, P. S. Sahni, and G. S. Grest, Phys. Rev. B 28, 2693 (1983); P. S. Sahni, D. J. Srolovitz, G. S. Grest, M. P. Anderson, and S. A. Safran, ibid. 28, 2705 (1983); G. S. Grest, D. J. Srolovitz, and M. P. Anderson, Phys. Rev. Lett. 52, 1321 (1984); G. S. Grest and D. J. Srolovitz, Phys. Rev. B 30, 5150 (1984).

${ }^{4}$ A. Sadiq and K. Binder, Phys. Rev. Lett. 51, 674 (1983); J. Stat. Phys. 35, 517 (1984).

${ }^{5}$ D. Turnbull, Trans. AIME 191, 661 (1952).

${ }^{6}$ I. M. Lifshitz, Zh. Eksp. Teor. Fiz. 42, 1354 (1962) [Sov. Phys. JETP 15, 939 (1962)].

${ }^{7}$ S. M. Allen and J. W. Cahn, Acta Metall. 27, 1085 (1979).

${ }^{8}$ O. G. Mouritsen, Phys. Rev. B 28, 3150 (1983); 31, 2613 (1985); 32, 1632 (1985); see also, K. Kaski, S. Kumar, J. D. Gunton, and P. A. Rikvold, ibid. 29, 4420 (1984).

${ }^{9}$ O. G. Mouritsen, Phys. Rev. Lett. 56, 850 (1986).

${ }^{10} \mathrm{We}$ assume here that the system is quenched to a temperature at which defect (vortex) motion is still possible. In continuous-spin models, putting the system on a lattice may give rise to an energy barrier for defect motion. In quenches of the system to zero temperature, as is done in some of these computer simulation, the growth kinetics may then be slower than predicted by the Lifshitz-Allen-Cahn law. Lowtemperature pinning of growth by vortices was observed in clock models by G. S. Grest and D. J. Srolovitz, Phys. Rev. B 30, 6535 (1984). Such pinning is, of course, absent for defect motion in liquid crystals at finite temperatures; see P. E. Cladis, W. van Saarloos, P. L. Finn, and A. R. Kortan, Phys. Rev. Lett. 58, 222 (1987).

${ }^{11}$ A. Milchev, K. Binder, and D. W. Heermann, Z. Phys. B 63, 521 (1986).

${ }^{12}$ R. Pindak, C. Y. Young, R. B. Meyer, and N. A. Clark, Phys. Rev. Lett. 45, 1193 (1980).

${ }^{13} \mathrm{We}$ stress that this independence of $c$ on the interface width is only true within the AC theory, and, close to $T_{c}$, if the dynamic critical exponent $z$ is equal to 2 (see Ref. 11).

${ }^{14}$ P. G. de Gennes, The Physics of Liquid Crystals (Oxford Univ. Press, Oxford, 1974).

${ }^{15}$ Without loss of generality, we have taken $P=1$ in Mouritsen's model and performed a gauge transformation by rotating the spins in every other row by the angle $\pi$. This makes the first term ferromagnetic.

${ }^{16}$ This transition is reminiscent of the transition between continuous and discontinuous dislocations discussed by $\mathbf{M}$. $\mathbf{H}$. Grabow and G. H. Gilmer, in Layered Structures and Epitaxy, Materials Research Society Proceedings, edited by $\mathrm{J}$. M. Gibson, G. C. Osbourn, and R. M. Tromp (NorthHolland, Amsterdam, 1986), Vol. 56. 